\title{
LA FORMACIÓN DE GUÍAS DE TURISMO DE MONTAÑA Y ALTA MONTAÑA: EL CASO CHILENO
}

\author{
Autores \\ Pablo Rebolledo Dujisin \\ Planificador y Gestor Turístico \\ Msc $\odot$ Desarrollo Regional y Medio Ambiente \\ Universidad de Valparaíso \\ Chile \\ rebolledo.dujisin@gmail.com \\ Rodrigo Fica Pérez \\ Ingeniero Civil Industrial \\ Pontificia Universidad Católica de Chile \\ Chile \\ En nuestros días, dentro de las ciudades, pocas cosas sobreviven, \\ la noche no existe ya, ni el frío, ni el viento ni las estrellas, todo \\ está neutralizado. \\ Gastón Rebuffat - La Montagne est mon Domaine
}

\section{RESUMEN}

La actividad de turismo de montaña se ha ido desarrollando en el mundo como es la tendencia del turismo de intereses especiales, sin embargo en Chile, los recursos humanos para este tipo de actividad no han podido ser regularizados ni normalizados; diferentes intentos por parte de instituciones han intentado subsanar esta situación, sin embargo hasta ahora no se ha logrado de forma efectiva ni mucho menos eficiente.

Las exigencias del turista demandan hoy un sistema de estandarización para los guías de montaña que sea aplicable y que permitan el crecimiento y desarrollo de la actividad.

Palabras Clave: Guías de Montaña, Turismo de Montaña, Formación para Guías, Regulación de Montaña. 


\title{
THE GUIDES FORMATION FOR MOUNTAIN TOURISM: THE CHILEAN CASE
}

\author{
Authors \\ Pablo Rebolledo Dujisin \\ Tourism Planner and Manager \\ Msc $\subset$ Environment and Regional Development \\ Universidad de Valparaíso \\ Chile \\ rebolledo.dujisin@gmail.com

\section{Rodrigo Fica Pérez} \\ Industrial Engineer \\ Pontificia Universidad Católica de Chile \\ Chile
}

Nowadays, inside the cities, few things survive, the night does not exist anymore, neither the cold, nor the wind nor the stars, everything has been neutralized.

Gastón Rebuffat - La Montagne est mon Domaine

\begin{abstract}
in tourism activity has been increasing in the world as is the tendency of Special Interest Tourism, nevertheless in Chile, there is neither regularization nor normalization for human resources operating this kind of tourism; different attempts from different institutions have tried to improve this situation, however, until now no one has achieved it in a neither effective nor efficient way.
\end{abstract}

The modern tourist demands a standardization system for mountain guides which should also be applicable and a facilitator for the activities both growth and development.

Key words: Mountain Guides, Mountain Tourism, Guides Formation, Mountain Regulation. 


\section{INTRODUCCIÓN}

¿Cómo se podría medir el grado de desarrollo que tiene la actividad del turismo en montaña en Chile? Es una pregunta que hasta el día de hoy no tiene respuesta pues, al igual que en muchos países de la región, no existen datos reales que permitan diagnosticar de forma realista la situación, generar tendencias o explicar con seguridad la situación actual y proyectada del turismo de montaña y alta montaña en el país.

Por otro lado, se puede afirmar que (quizás como vara para medir el desarrollo de la actividad turística en montaña) muchos de los países que poseen cadenas montañosas, especialmente Europa, el trabajo de Guía de Montaña ya no es un oficio, sino una carrera profesional, que involucra varios años de formación, un rendimiento físico-técnico de primer nivel y el cumplimiento de estándares severos. Sin embargo la situación en Chile difiere de la antes descrita pues hoy, por temas de demanda, oferta, interés o conveniencia, Chile carece de un sistema regulatorio efectivo y eficiente para con el turismo de montaña.

Primero que nada se debe aclarar, en pocas palabras que, para que se necesiten guías, se requiere que exista gente que necesite y quiera ser guiada. De perogrullo, pero necesario recalcar, puesto que a diferencia de los montañistas que hacen actividad por y para sí, aquí la viabilidad del trabajo de Guía dependerá de la existencia de una demanda, es así como esta actividad cumple, como cualquier otra actividad comercial, con las leyes de oferta y demanda.

Mientras no existan turistas interesados en contratar el servicio de un profesional que los acompañe a la montaña, que les muestre el camino, vele por su seguridad y les provea de servicios, el oficio de Guía de Montaña en Chile seguirá siendo sólo un oficio.

Se presenta a continuación un ensayo que se centra en dos aspectos fundamentales, primero que nada el desarrollo de la actividad guiada de montaña y segundo un resumen de la situación en Chile y las regulaciones que han ido y venido con el tiempo.

\section{Antecedentes: La Cordillera de los Andes}

Las montañas han sido buscadas como destinos turísticos desde hace mucho tiempo, sin embargo hasta hace poco habían permanecido vedadas exclusivamente para los montañistas: gente que busca aventura de forma activa e independiente y que no se considera a sí mismo como turista; la situación en la actualidad ha cambiado, hoy la 
frontera entre la actividad deportiva de montaña y el turismo no es tan clara como hace unos años atrás (Beedie y Hudson, 2003).

En el caso chileno es innegable la persistencia de la cordillera a lo largo del país, es así como un gran número de las actividades turísticas que se desarrollan hoy en día en Chile están relacionadas con la montaña; ya sea actividades de contemplación, esquí y sus derivados o el deporte de montaña en todas sus expresiones: alta montaña, escalada, media montaña, excursionismo, senderismo, travesías y todas las combinaciones de las antes mencionadas, como también aquellas que aprovechan las pendientes formadas por la poca distancia entre la cordillera y el mar, para realizar actividades como el rafting o el kayak de río.

Sin embargo se debe tener algunos antecedentes de ésta: La cordillera recorre el continente sudamericano en toda su extensión, desde parte de Venezuela hasta Chile, donde su influencia es directa en cuanto su presencia es imponente en todo el territorio; con una altitud media de 4.000 m.s.n.m. su altitud máxima se encuentra en Argentina siendo representada por el monte Aconcagua, con 6.962 m.s.n.m. En el caso chileno, el punto más alto se encuentra en la III Región, el volcán Ojos del Salado alcanza los 6.893 metros sobre el nivel del mar; dicho esto, se debe aclarar que, en ningún caso (como es creencia popular) la cordillera de Los Andes es la más alta del mundo, pues es superada, al menos por el Himalaya, Karakoram y Tian-Shan, donde se encuentran altitudes sobre los ocho mil metros; esto es importante de señalar, pues en términos puros de turismo de montaña, los Andes chilenos no son, como la creencia popular dice, extraordinarios ni mucho menos "únicos", lo que se traduce, entre otras cosas, en que la demanda por turismo de montaña no es cautiva como en el caso del Himalaya.

Debido a la inexistencia de datos fidedignos no se puede precisar la cantidad de turistas que acceden a las montañas chilenas, ni tampoco cual es la más demandada turísticamente, sin embargo la percepción podría indicarnos que, entre las más buscadas por los turistas, tanto chilenos como extranjeros, se encuentran el Ojos del Salado, por ser la más alta del territorio como se mencionó antes o el cerro El Plomo en la cordillera de Santiago por su representatividad en el paisaje y su cercanía con esta metrópolis, a lo anterior se le debe sumar el Volcán Villarrica que mantiene un sistema turístico operativo desde la localidad de Pucón (IX Región) y que registra gran cantidad de movimiento turístico, siendo, posiblemente, la más visitada en Chile.

\section{El Guía de Montaña}

El Guía de montaña, como actor del turismo, es el operador en ambientes de montaña y alta montaña; la labor del guía, en este caso, consiste en conectar los servicios, aprovechar sus posibilidades y facilitar al turista la experiencia, el disfrute 
de los recursos y de los servicios a su disposición. Es el touroperador quien en realidad mantiene el contacto con el cliente (Ejarque, 2005).

Definir las funciones de un guía de montaña es siempre una labor compleja, hoy existe, en Chile un amplio vacío en cuanto a las funciones, tareas, sueldos y responsabilidades que tienen los guías en la montaña, trayendo las consecuencias del desarrollo improvisado de una profesión.

Es el ambiente de montaña, peligroso e indomable el que, dentro de un contexto de aventura, genera el entusiasmo para realizar una actividad donde existen riesgos incontrolables (Beedie y Hudson, 2003); el guía debe encontrar el punto de balance entre lo que es peligroso o "emocionante" y lo que es seguro, quizás "aburrido". Considerando estos factores de importancia al momento de llevar grupos humanos a la montaña; entonces se tiene en una mano lo que representa excitación, placer, interés, espontaneidad, disfrute y libertad; y en la otra lo que representa demasiada disciplina llevando a un régimen monótono o la rigidez estéril que viene desde la sobre preparación y sobre planificación. (Langmuir, 1984)

El guía debe entonces, estar siempre consiente si la balanza se inclina hacia un lado o hacia otro ya sea por su propia arrogancia o por su ansiosa falta de confianza o carencia de experiencia. (Langmuir, 1984)

En este contexto, se debe mencionar que, a diferencia de la gran mayoría de las actividades guiadas, en el turismo de montaña, los clientes deben funcionar como "montañistas" y no simplemente como espectadores, son partícipes de la actividad y co-responsables de su seguridad, tener conocimientos técnicos y una condición física de excelencia; se presenta entonces una dualidad entre turista y aspirante a montañista, puesto que, a medida que los clientes se hacen fieles a la actividad, ganan habilidades y capacidad por "hacer" montañismo (Beedie y Hudson, 2003), exigiendo así altas habilidades técnicas, físicas y conocimientos por parte del guía de montaña.

El escenario es claro: la importancia de contar con guías capacitados, responsables y altamente técnicos es indiscutible, siendo la formación y la experiencia el único camino realista y eficaz para que el turismo pueda generar mejoras tangibles en la competitividad e incremento de la productividad (López y García, 1998); es así como para apuntar hacia el desarrollo turístico sostenible la calidad de los recursos humanos, en este caso los guías de montaña, tiene un papel decisivo y se precisa un importante esfuerzo de formación (Szmulewicz, 2000). Puesto que el guía, moderno y profesional, ya no se encarga únicamente de llegar a una persona de un lugar a otro, de encontrar el sendero y el campamento, sino que debe ser capaz de conocer la historia, la geografía, mantener la moral del grupo y conocer técnicas de resolución de conflictos y de dinámica 
grupal; su labor es proveer seguridad, mediación e interpretación (Cohen, et al, 2002)

\section{La formación del personal del Turismo}

Si se considera la industria turística como aquella que pretende ofrecer al turista diferentes experiencias y emociones, ciertamente, todos los elementos que rodean la experiencia turística del viajero, y que va a determinar su nivel de satisfacción, están directamente relacionados con la labor desempeñada por los profesionales del sector; el componente humano juega, entonces, un papel fundamental para el desarrollo del turismo (Lillo, Ramón y Sevilla, 2007). Los Recursos Humanos son hoy el personal que está en directo contacto con el turista, siendo este uno de los principales abanderados del turismo en un destino.

En este sentido, Bote (1995) asegura que la formación en turismo debe constituir un eje fundamental del modelo de desarrollo cualitativo propugnado desde el ámbito académico y que permita otorgarle calidad al servicio dependiente del factor humano. Podemos asegurar entonces que existe una relación directa entre formación en turismo y calidad de la experiencia turística.

La importancia estratégica de este recurso, el capital humano como capacidad distintiva, nos conduce a plantear la necesidad de diseñar políticas de educación y formación de calidad en turismo (Lillo, Ramón y Sevilla, 2007), políticas que hasta el día de hoy no han sido implementadas para los guías de montaña, quienes pasan desde aquellos con una formación de excelencia hasta aquellos que nunca han recibido la más mínima capacitación ni deportiva ni técnica ni mucho menos de calidad en turismo.

\section{Los primeros pasos formativos en Chile: La Certificación de Guía ENAM}

Dado que las estadísticas y registros se hacen escasas o inexistentes, no se puede afirmar a ciencia cierta la historia que han recorrido los procesos de certificación de Guías de Montaña en Chile: Uno de los primeros, sino el primero, fue el esfuerzo que realizó, a mediados de la década del '90, la Escuela Nacional de Montaña (ENAM) para formar guías en Chile.

Esta institución ya venía realizando labores preliminares al respecto, puesto que, en el Chile de 20 años atrás, era la única institución que podría haber tomado un rol así frente a los deportistas de montañas y aficionados guías y determinar cuáles de ellos sí tenían las competencias necesarias para desempeñarse laboralmente en la montaña, confundiendo muchas veces los roles de instructor de montaña y guía de montaña.

Tras varios procesos de re-certificación y visitas a terreno a lugares claves la 
ENAM asumió la formación de los futuros guías de montaña. Inquietud que desembocó en 1996, bajo el mandato de Vicente Sigl y la dirección técnica de Claudio Gálvez (QEPD), en la creación del Primer Curso para Guías de Excursionismo, Montaña y Alta Montaña de Chile.

Este fue claramente un esfuerzo muy adelantado para la época (al menos en Chile) y consistía en un curso de cuatro semestres de duración con un plan de estudio teórico-práctico, que abarcaba tópicos como: Primeros Auxilios, Radiocomunicaciones, SNASPE, Vida Silvestre, Alimentación de montaña e Inglés para el turismo, a los cuales se agregaban los específicos propios del montañismo (nudos, escalada, auto-rescate, etc.), enseñados, practicados y evaluados constantemente en la más de una docena de salidas a terreno que se debían realizar.

Sin embargo, al momento de llevar a la práctica dicho programa, surgieron una serie de problemas que terminaros por hacer inviable la iniciativa, entre estos destaca el hecho que prácticamente se aceptó que cualquier persona lo pudiera tomar.

Inicialmente, debido al obvio foco de profesionalismo del curso, quienes querían participar debían tener un currículum deportivo abultado en cantidad y calidad, sumado al requisito de pasar varias pruebas técnicas en una salida especialmente dedicada a seleccionar los mejores.

Pero para hacerlo viable económicamente, se necesitaba que una cantidad de alumnos que rondaba las veinte personas, que pasaran el proceso de selección y que, luego, estuvieran en condiciones de pagar el; bajo estas condiciones el curso de formación se hizo inviable, ante lo cual la Escuela Nacional de Montaña tomó la decisión de bajar los requisitos de ingreso significando una baja, también, en la calidad del curso generándose grupos altamente heterogéneos en su formación deportiva en montaña; sin embargo el curso se dictó de forma periódica con altos y bajos pero se convirtió en un real aporte para la formación de guías en Chile.

Lamentablemente por azares del destino, en un corto periodo de tiempo el núcleo de la ENAM se desintegró, pues murieron dos de sus más expertos instructores: Sergio Zárate murió en un accidente en los Rodados de San Gabriel y Max Bombardiere en un extraño incidente en Punta de Tralca. Finalmente y como si fuese poco Claudio Gálvez, en ese tiempo director de la Escuela, falleció al sufrir una caída bajando de la cumbre del Gasherbrum I. Esto trajo consigo la desestabilización del sistema que terminó por significar el cierre de dicho curso.

En términos de formación de guías de montaña se produjo, entonces, un vacío temporal; recordando siempre que este ensayo trata de la formación de guías de montaña 
y no de guías de turismo aventura ni técnicos en esta disciplina.

\section{Proceso de Normalización}

INN, acrónimo para Instituto Nacional de Normalización, es una fundación técnica de derecho privado sin fines de lucro, creada por CORFO, que se dedica a fomentar el uso de normas técnicas en el país (INN, 2009).

En conjunto con otras instituciones, entre ellas SERNATUR, a partir del 2004 el INN lideró el procedimiento de normalización de las actividades de turismo en montaña, buscando un conjunto razonable de requisitos mínimos que deberían cumplir los servicios guiados de montañismos. El esfuerzo fue macro y abarcó prácticamente todas las actividades relacionadas con el montañismo: campismo, senderismo, excursionismo, barranquismo, esquí fuera de pista, escalada deportiva, escalada en roca, escalada en hielo y otros tantos.

El proceso, en cuanto al ámbito de montaña se refiere, fue dirigido por Ramona Villalón, coordinadora general del proyecto Sistema Normativo y Calidad Turística, en reuniones abiertas y ampliadas donde se hizo una invitación extensa a participar del proceso. Es decir se invitó a toda institución que podría llegar a tener relación tanto con el turismo como con el deporte de montaña; ya sea esporádica o consistentemente participaron la Asociación de Guías de Alta Montaña AGAM, Escuela Nacional de Montaña ENAM, la Federación de Andinismo de Chile FEACH, La Asociación de Agencias de Turismo de Aventura CATA, Cuerpo de Socorro Andino CSA, la Federación de Esquí, varias agencias de turismo aventura, las empresas de muros de escalada, los clubes deportivos, las asociaciones deportivas, deportistas de montaña y los guías de montaña.

Como es de esperarse las normas no concitaron unanimidad, pero, puesto que los expertos fueron consultados y las normas van en la misma línea que otras similares en el extranjero (como la certificación UIAGM) se pueden considerar como un fuerte incentivo a la regularización de la actividad con una excelente base teórica, que, sin embargo, a la fecha de la presentación de este ensayo, no tenía reconocimiento en su aplicación entre los guías de montaña.

\section{Los Gremios}

Difícil es catastrar completamente y mantener actualizada la cantidad y calidad de las asociaciones de guías de montaña que hay en Chile, puesto que, en términos reales, muchas de ellas existen sin conducción ni orgánica y con muy poca capacidad de convocatoria. 
Dentro de las asociaciones con mayor presencia hoy en el territorio nacional se encuentran la Asociación de Guías de Montaña de Pucón, la Asociación Gremial de Guías de Turismo de Última Esperanza (AGGTUE) y la Asociación de Guías de Alta Montaña (AGAM, con base en Santiago). Estas tres son las únicas en Chile que han logrado, influenciar su entorno; sin embargo, históricamente estas instituciones en Chile han sido débiles y están lejos del grado de organización y desarrollo que tienen las existentes en países como Argentina, Perú y Bolivia; para efectos del trabajo de guía de montaña en Chile, hoy la institucionalidad gremial es inexistente: nunca ha sido capaz de llegar al Poder Legislativo para la generación de leyes en su beneficio, no ha traído ningún estándar internacional a Chile, desarrolla un trabajo en el tiempo que es discontinuo y descoordinado, ha dejado que surjan diversos estándares contradictorios (CATA, Federación, ENAM, INN), no tiene presencia en los medios de comunicación y raramente es considerada como un interlocutor valido.

\section{Normas Internacionales}

UIAGM es acrónimo de "Unión Internationale des Associations de Guides de Montagnes" (en inglés, IFMGA), organización fundada en 1965-1966 por las asociaciones de guías de Italia, Francia, Austria y Suiza, con la intención de promover y facilitar la práctica de dicha profesión alrededor del mundo. Un colectivo importante, con reconocimiento y sinónimo de excelencia, que representa hoy en día a más de 6.000 guías y que, dicho sea de paso, no tiene relación ni depende de la UIAA. (UIAGM, 2009)

Una de sus tareas básicas fue la de establecer un estándar que certificara profesionales para trabajar en cualquier montaña o cordillera del planeta (conociéndola o no). Una norma que permitiera diferenciar al guía experto del aficionado, popularmente conocida como "la certificación UIAGM".

Esta “certificación" es hoy en día uno de los estándares más estrictos, requiriendo entrenamiento, experiencia, técnica y conocimientos específicos, lo que, en promedio toma una dedicación completa de entre tres a cinco años.

Este estándar ha intentado ser introducido en Chile en variadas ocasiones, con, al menos, tres intentos fallidos. La última tentativa se desarrolló el año 2008, en un proceso patrocinado por Argentina; sin embargo la cantidad de chilenos que cumplían los requisitos mínimos para participar y que a la vez se vieron interesados en someterse al proceso no pasaron de diez; cuando, para hacer viable el proceso financiera y educacionalmente, se necesitaban por lo menos veinte.

Se debe mencionar, entonces, que este estándar ya funciona hace varios anos 
en países como Argentina, Perú y Bolivia donde existe una demanda por guías y a la vez por programas de formación en estas profesiones.

\section{El estado actual de los Guías de Montaña en Chile}

Primero que nada se debe comprender, después de lo antes expuesto que ningún programa de formación en montaña puede enseñar cómo ser infalible ni tampoco puede (ni podrá) otorgar una serie de reglas y procedimientos que funcionen en cada una de las ocasiones que pueden ocurrir en montaña; solo pueden entregar una serie de herramientas que funcionan en ya establecidos escenarios, la aplicación de éstas en otras circunstancias dependen exclusivamente de la experiencia del guía y de sus habilidades personales (Langmuir, 1984). Sin embargo, en la actualidad es fundamental que la preparación y el nivel de profesionalidad del personal de un destino sea elevado. Si la profesionalidad y la formación no existen, un destino se ve avocado a la constante improvisación y a una falta de visión estratégica que repercutirá en el desarrollo de todo el sistema turístico. (Ejarque, 2005)

Los procesos antes mencionados son reales y existen en diferentes países, a la vez es un hecho que en Chile aun no se ha podido implementar de forma eficiente ningún sistema que regularice definitivamente la actividad.

En general esta situación se puede atribuir al tipo de mercado en el que se encuentra compitiendo Chile; en pocas palabras, los estándares son caros y hoy la poca demanda para ascensiones de montaña guiadas no justifican la inversión puesto que los clientes no están dispuestos a pagar una suma de dinero considerable para realizar la inversión en capacitación, entrenamiento, tecnificación y equipo.

En Chile uno de las montañas más visitadas es el Cerro El Plomo, con cerca de mil visitantes al año (Wevar, 2007), en cambio solo el Aconcagua, en Argentina, recibe más de siete mil visitas anuales (Parque Provincial Aconcagua, 2009). Esto hace que hoy, con la poca demanda que existe, los guías no se interesen en certificarse ni homologarse, puesto que en la práctica una certificación internacional corresponde a un desembolse de dinero que hoy no es necesario hacer para desempeñarse como guía. 


\section{CONCLUSIONES}

Todo indica que el turismo de montaña continuará creciendo a nivel mundial y que los riesgos inherentes a la actividad tenderán a disminuir en cuanto se siga desarrollando la técnica, tecnología, conocimientos y regulaciones; sin embargo, se debe comprender que la actividad de montaña es de por si peligrosa y que nunca (al menos en un escenario imaginable) los riesgos y peligros podrán ser controlados del todo, donde el rol del guía, como líder y encargado de seguridad, es uno de los factores clave para el crecimiento de la actividad.

Las empresas de turismo de montaña han generado y creado paquetes que requieren ser ejecutados por recursos humanos de excelencia: primero, una empresa necesita montañistas, gente con experiencia en terreno, actividad deportiva destacada y ascensiones que lo hayan llevado a tomar decisiones importantes durante su desempeño como deportista, y segundo, guías preferentemente calificados, con estudios y formación en escuelas establecidas que no permitan el desarrollo de "malas mañas" o conocimientos erróneos acerca de la actividad de montaña (el ejemplo más típico del error de montañista sin formación es creer que las bebidas alcohólicas deben consumirse en caso de sentir frío).

Es claro que la industria turística hoy en Chile presenta una falencia importante en cuanto a Guías de Montaña se refiere; todos los estudios y la experiencia apuntan a que un servicio regulado, estandarizado y normalizado es base para una experiencia turística satisfactoria, en especial en un turismo tan delicado como el de montaña, donde accidentes mortales ocurren año a año, tanto en Chile como en sus países vecinos y en otros que ya poseen complejos sistemas normativos y cursos de capacitación de excelencia.

Ahora bien, esfuerzos como el de ENAM, CATA, INN y otros han dado lineamientos para intentar clarificar el escenario para los guías, esfuerzos que sin embargo hoy carecen de una base firme que toda industria necesita: la demanda. Es quizás la lejanía de Chile, quizás su poco desarrollo en infraestructura de montaña o las definitivamente más bajas ventajas comparativas con otras cadenas montañosas en el mundo lo que han llevado a que Chile demande muy poco por guías de turismo de montaña, sea un país caro en términos de turismo de montaña y tenga guías poco preparados.

Si es el factor humano uno de los más importantes elementos de la cadena productiva turística entonces ¿no se requerirá un esfuerzo aun mayor que la mera creación de las normas y estándares para guías de montaña? Al parecer se hace necesario un esfuerzo a nivel gubernamental que apoye y exija la certificación de los guías teniendo en 
consideración no sólo el escenario ideal que contemple guías muy preparados y capaces sino también las condiciones del mercado que regulan la actividad, donde el guía se vea incentivado a normalizarse no porque el precio del proceso de certificación sea bajo, sino porque recibirá beneficios tangibles para desarrollar su profesión de forma sustentable.

Bajo el escenario actual serán muy pocos los guías que deseen certificarse por medio de algunos de los varios estándares nacionales, a sabiendas de que sus ingresos no aumentarán y sus empleadores no necesariamente les darán más empleo. Por lo que es necesario un sistema que incentive tanto a guías como empresarios y demandantes de este turismo realizado por un grupo muy selecto y especializado de visitantes.

\section{BIBLIOGRAFÍA}

》 Beedie, P. Hudson, S. (2003). Emergence of Mountain-Based Adventure Tourism. Annals of Tourism Research, Vol 30, N³. Gran Bretaña.

$\gg$ Bote, V. (1995) Estructura y desarrollo del turismo en España: hacia un cambio cualitativo y más responsable. Congreso de Economía del Turismo, Colegio de Economistas de las Palmas de Gran Canaria.

$\gg$ Cohen, E. Cohen, E. Ifergan, M. (2002). A New Paradigm in Guiding, the Madrich as a Role Model. Annals of Tourism Research, Vol 29, N 4. Gran Bretaña.

》 Ejarque, J. (2005). Destinos Turísticos de Éxito; Diseño, creación, gestión y marketing. Ediciones Pirámide, Madrid.

\Instituto Nacional de Normalización. (2009). El INN. (En Línea) [Disponible en la Web en: www3.inn.cl/inn/portada/index.php]. Citado en 10 de Julio de 2009.

$\gg$ Langmuir, E. (1984). Mountain Craft and Leadership. Editado por The Scottish Sport Council. Edimburgo.

\ Lillo, A. Ramón, A. Sevilla, M. (2007). El Capital Humano Como Factor Estratégico Para la Competitividad del Sector Turístico. En Cuadernos de Turismo, $N^{\circ} 19$. Universidad de Murcia, España

》 López, P. García, J. Hernández, J. (1998). Acceso Laboral y Formación Turística. En Cuadernos de Turismo, $\mathrm{N}^{\circ}$ 1. Escuela Universitaria de Turismo de Murcia, España.

》 Parque Provincial Aconcagua. (2009). Tarifas y Estadísticas. (En Línea) [Disponible en la Web en: www.aconcagua.mendoza.gov.ar/estadisticas.pdf]. Citado en 16 de Julio de 2009.

\Szmulewicz, P. (2000). Calidad de Recursos Humanos Para un Turismo Rural 
Sustentable. En Gestión Turística. №5 Edición Especial. Universidad Austral de Chile, Valdivia.

》 Unión Internationale des Associations de Guides de Montagnes. (2009). La UIAGM. (En Línea) [Disponible en la Web en: www.ivbv.info/es/la-uiagm/ presentacion.html]. Citado en 18 de Julio de 2009.

$\gg$ Wevar, R. (2007) Contabilización de montañistas que visitan el Cerro El Plomo y sus apreciaciones con respecto a éste. Informe de Práctica Profesional para Centro Cordillera, Municipalidad de Lo Barnechea, Santiago.

Recibido: 17/08/2009

Aprobado:20/09/2010

Arbitrado anonimamente 